\title{
Assessment of the Diagnostic Ability of Four Detection Methods Using Three Sample Types of COVID-19 Patients
}

\section{OPEN ACCESS}

Edited by:

Rodolfo García-Contreras, National Autonomous University of Mexico, Mexico

Reviewed by:

Elmira Esmaeilzadeh, Brigham and Women's Hospital and Harvard Medical School, United States

Hector Quezada,

Hospital Infantil de México Federico

Gómez, Mexico

Rafaela González Montelongo, Instituto Tecnológico y de Energías Renovables (ITER), Spain

*Correspondence: Yu Chen

chenyuzy@zju.edu.cn

${ }^{\text {t}}$ These authors have contributed equally to this work

Specialty section: This article was submitted to Clinical Microbiology, a section of the journal Frontiers in Cellular and Infection Microbiology

Received: 26 March 2021 Accepted: 19 May 2021 Published: 07 June 2021

\section{Citation:}

Yu F, Xie G, Zheng S, Han D, Bao J,

Zhang $D$, Feng $B$, Wang $Q$, Zou $Q$,

Wang $R$, Yang $X$, Chen $W$, Lou $B$ and

Chen $Y$ (2021) Assessment of the

Diagnostic Ability of Four Detection

Methods Using Three Sample

Types of COVID-19 Patients.

Front. Cell. Infect. Microbiol. 11:685640.

doi: 10.3389/fcimb.2021.685640

\begin{abstract}
Fei $\mathrm{Yu}^{1,2,3 \dagger}$, Guoliang Xie ${ }^{1,2,3 t}$, Shufa Zheng ${ }^{1,2,3 \dagger}$, Dongsheng Han ${ }^{1,2,3}$, Jiaqi Bao ${ }^{1,2,3}$, Dan Zhang 1,2,3, Baihuan Feng 1,2,3, Qi Wang ${ }^{1,2,3}$, Qianda Zou 1,2,3, Ruonan Wang 1,2,3, Xianzhi Yang ${ }^{1,2,3}$, Weizhen Chen ${ }^{1,2,3}$, Bin Lou ${ }^{1,2,3}$ and Yu Chen ${ }^{1,2,3,4 *}$

${ }^{1}$ Department of Laboratory Medicine, The First Affiliated Hospital, Zhejiang University School of Medicine, Hangzhou, China, ${ }^{2}$ Key Laboratory of Clinical In Vitro Diagnostic Techniques of Zhejiang Province, Hangzhou, China, ${ }^{3}$ Institute of Laboratory Medicine, Zhejiang University, Hangzhou, China, ${ }^{4}$ State Key Laboratory for Diagnosis and Treatment of Infectious Diseases, National Clinical Research Center for Infectious Diseases, Collaborative Innovation Center for Diagnosis and Treatment of Infectious Diseases, The First Affiliated Hospital, Zhejiang University School of Medicine, Hangzhou, China
\end{abstract}

Background: Viral nucleic acid detection is considered the gold standard for the diagnosis of coronavirus disease 2019 (COVID-19), which is caused by SARS-CoV-2 infection. However, unsuitable sample types and laboratory detection kits/methods lead to misdiagnosis, which delays the prevention and control of the pandemic.

Methods: We compared four nucleic acid detection methods [two kinds of reverse transcription polymerase chain reactions (RT-PCR A: ORF1ab and N testing; RT-PCRB: only ORF1ab testing), reverse transcription recombinase aided amplification (RT-RAA) and droplet digital RT-PCR (dd-RT-PCR)] using 404 samples of 72 hospitalized COVID-19 patients, including oropharyngeal swab (OPS), nasopharyngeal swabs (NPS) and saliva after deep cough, to evaluate the best sample type and method for SARS-CoV-2 detection.

Results: Among the four methods, dd-RT-PCR exhibited the highest positivity rate (93.0\%), followed by RT-PCR B (91.2\%) and RT-RAA (91.2\%), while the positivity rate of RT-PCR A was only 71.9\%. The viral load in OPS [24.90 copies/test (IQR 15.58-129.85)] was significantly lower than that in saliva [292.30 copies/test (IQR 20.20-8628.55)] and NPS [274.40 copies/test (IQR 33.10-2836.45)]. In addition, if OPS samples were tested alone by RT-PCR A, only $21.4 \%$ of the COVID-19 patients would be considered positive. The accuracy of all methods reached nearly $100 \%$ when saliva and NPS samples from the same patient were tested simultaneously.

Conclusions: SARS-CoV-2 nucleic acid detection methods should be fully evaluated before use. High-positivity rate methods such as RT-RAA and dd-RT-PCR should be considered when possible. Furthermore, saliva after deep cough and NPS can greatly improve the accuracy of the diagnosis, and testing OPS alone is not recommended.

Keywords: COVID-19, SARS-CoV-2, sample type, saliva, dd-RT-PCR, RT-RAA 


\section{INTRODUCTION}

Since the first emerging in late 2019, coronavirus disease 2019 (COVID-19) has caused a worldwide pandemic, with more than 157 million confirmed cases and 3 million deaths (WHO, 2021). Early diagnosis and treatment of suspected patients is the key to effectively control COVID-19 (Chu et al., 2020; Li et al., 2020). Currently, viral nucleic acid detection is still the most effective method to confirm SARS-CoV-2 infection. A variety of detection methods based on specific SARS-CoV-2 nucleotide sequences have been rapidly developed and used as emergency applications in the laboratory. To date, National Medical Products Administration (NMPA China) has approved 22 SARS-CoV-2 nucleic acid detection reagents, most of which are reverse transcription polymerase chain reaction (RT-PCR) methods. In addition, some other detection techniques are waiting for approval, such as reverse transcription recombinase aided amplification (RT-RAA) method and droplet digital RT-PCR (dd-RT-PCR) method. RAA is a new type of nucleic acid amplification technology developed in recent years, and it works using four enzymes (UvsX, UvsY, SSB, and polymerase) at a constant temperature of $37 \sim 42^{\circ} \mathrm{C}$ (Zhang et al., 2017). Compared to RT-PCR, RT-RAA based assay is faster, simpler and no need for fluorescent quantitative PCR instruments (Wang J. et al., 2020). The dd-RT-PCR is a new method of digital PCR that enables the absolute quantification of nucleic acid without the use of calibration curves (Hindson et al., 2011). Meanwhile, it has been reported that the positivity rate or sensitivity of initial RT-PCR result is compared to result after repeated tests of RT-PCR as reference standard methods, with a range of 51.25\% to $94.6 \%$ (Axell-House et al., 2020). Reasons for the false negatives of initial RT-PCR may include insensitive nucleic acid detection kits, variations in the accuracies of different tests, low initial viral load or improper clinical sampling (Fan et al., 2020; Fang et al., 2020). Thus, multiple samples and repeated testing may be required to diagnose patients who are infected with SARS-CoV-2.

In this study, we compared two RT-PCR reagents approved by the NMPA China, an RT-RAA reagent, and a dd-RT-PCR reagent, using multiple types of sample of hospitalized COVID19 patients, to evaluate the performance of different methods. Simultaneously, we compared the positivity rate of using three common sample types, namely oropharyngeal swab (OPS), nasopharyngeal swabs (NPS) and saliva after deep cough, to provide empirical evidence for the selection of suitable samples and methods in the diagnosis of SARS-CoV-2 infection.

\section{MATERIALS AND METHODS}

\section{Subjects}

This study enrolled a total of 72 COVID-19 patients, who were admitted to the First Affiliated Hospital, Zhejiang University School of Medicine, from $19^{\text {th }}$ Jan 2020 to $23^{\text {rd }}$ Feb 2020. All enrolled cases were confirmed to be infected by SARS-CoV-2 through multiple repetitions of RT-PCR detection (RT-PCR A:
BioGerm, Shanghai, China). COVID-19 patients were diagnosed according to the $6^{\text {th }}$ edition of the Guideline for Diagnosis and Treatment of SARS-CoV-2 issued by the National Health Commission of the People's Republic of China. The demographic information, medical comorbidities, date of symptom onset, symptoms and signs, progression and resolution of clinical illness during the hospitalization period were obtained from the clinical records. All data were reviewed by a trained team of physicians. This study was reviewed and approved by the Clinical Research Ethics Committee of the First Affiliated Hospital, Zhejiang University School of Medicine.

\section{Sample Collection}

Three high-concentration positive clinical samples were 5-fold serially diluted to $1: 78,125$ using normal saline to evaluate the limit of detection of each method, a total of 24 samples were obtained.

Excluding those 4 patients with tracheal intubation or coma, 68 hospitalized COVID-19 patients were included in study on $11^{\text {th }}$ Feb. These patients were all in the general isolation wards at the time of sampling. Before sampling, it was confirmed that the patient did not drink water, eat food, gargle or other similar behaviors within half an hour that might affect the sampling quality. The specific collection process is as follows: OPS were collected first, NPS was collected after a 15-minite' interval, and after another 15-minutes the patient was instructed to wear a mask and deep cough $3 \sim 5$ times before spitting saliva into a sterile container. OPS and NPS of each patient were obtained by experienced physicians using flocked swabs (FLOQSwabs, Copan Italia) and were transported in $3 \mathrm{~mL}$ universal transport medium (UTM, Copan Italia). Swabs were rotated and stayed for enough time to collect the fluid and epithelial cells. All samples were sent to the laboratory for testing within $1 \mathrm{~h}$ after sampling. A total of 204 samples were collected from the 68 patients.

Additionally, to dynamically evaluate four methods in different stages of disease, 12 of the 72 patients having wellpreserved saliva after deep cough were enrolled in this study. A total of 197 saliva samples from these 12 hospitalized patients were collected. Each patient provided 11 to 26 saliva samples. All samples were tested on the day of collection and were frozen at $-80^{\circ} \mathrm{C}$ after testing. The protocols of sample inclusion and testing are shown in Figures $\mathbf{1}$ and $\mathbf{3}$.

\section{Laboratory Testing}

For viscous saliva samples, an equal volume of $0.4 \mathrm{mg} / \mathrm{mL}$ protease $\mathrm{K}$ buffer was added to the samples before nucleic acid extraction. After that, the mixture was vortexed for $15 \mathrm{~s}$, left standing for $20 \mathrm{~min}$, vortexed for another $15 \mathrm{~s}$, and centrifuged at $13000 \mathrm{rpm}$ for $5 \mathrm{~min}$. Then supernatant was collected for viral RNA extraction. Viral RNAs of saliva supernatant, OPS and NPS were extracted using the MagNA Pure LC 2.0 (Roche, Basel, Switzerland). Finally, four methods were used to detect nucleic acid of SARS-CoV-2, including two RT-PCR kits (A: Cat. No. ZC-HX-201-2, BioGerm, Shanghai, China; B: Cat. No. MFG030010, BGI Genomics, Wuhan, China), an RT-RAA reagent (Cat. No. T00R01; Qitian, Wuxi, China), 


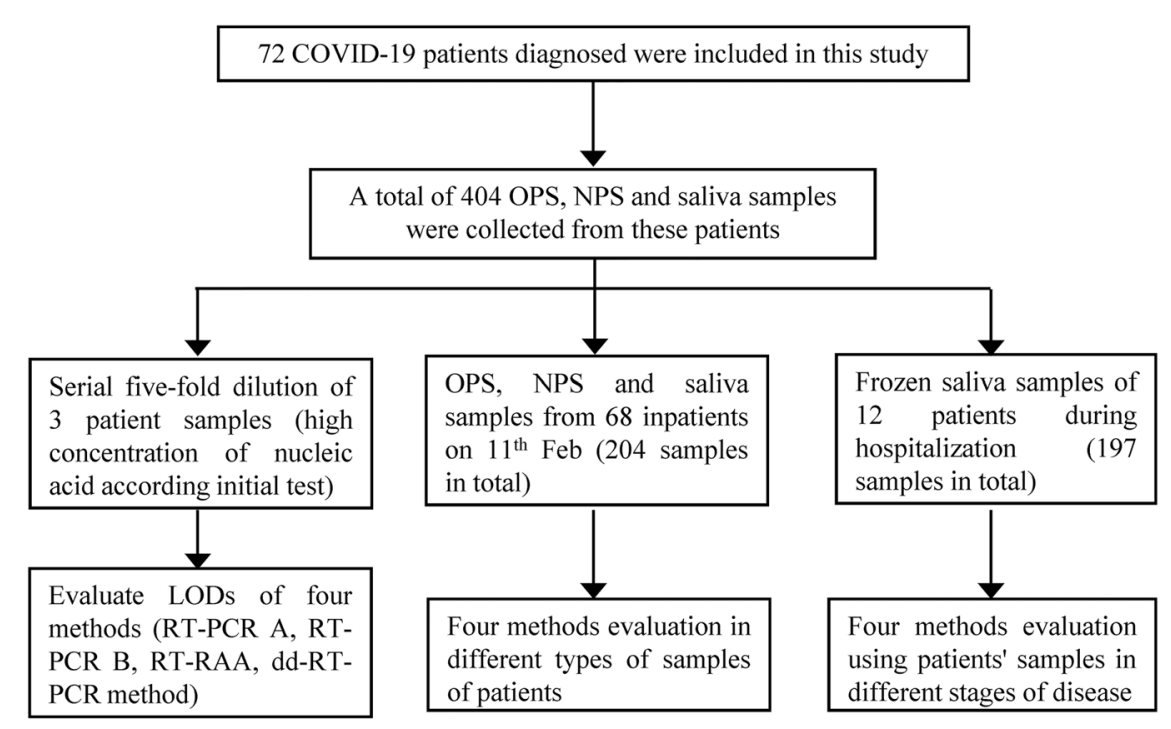

FIGURE 1 | Flowchart of patient/sample recruitment and study flow.

and a dd-RT-PCR reagent (Cat. No. 13444; TargetingOne, Beijing, China). The principles, targets, and result interpretation of the four methods are shown in Table 1. Operation and result assessment were conducted in accordance with the manufacturers' instructions. Negative and positive controls were setted for each test. In accordance with the guidelines of the Chinese Health Commission, all samples tested in this study were conducted in biosafety Level 2 laboratory. In addition, serially diluted samples were tested at the same time with three replicates, while the samples with suspected results were not tested again. The results were determined to be positive only when all three replicates were positive.

\section{Statistical Analysis}

Descriptive statistics included the mean with standard deviation (SD; for data with normal distribution), median with interquartile range (IQR; for data with skewed distribution), and proportion (\%). If any of the four methods showed a positive result for a sample, the specimen was classified as positive, and the positivity rate of each method was calculated. The Kruskal-Wallis test was used to evaluate the viral load among different sample types detected by dd-RT-PCR. A p value less than 0.05 (two-sided) was considered statistically significant. The statistical analysis was performed using either Prism 7.0 (GraphPad, La Jolla, CA, USA) or SPSS 17.0 (College Station, TX, USA) software.

TABLE 1 | Introduction of the 4 methods.

\begin{tabular}{|c|c|c|c|c|}
\hline Method & RT-PCR A & RT-PCR B & RT-RAA & dd-RT-PCR \\
\hline $\begin{array}{l}\text { Reaction } \\
\text { principle }\end{array}$ & $\begin{array}{l}\text { Quantitative reverse } \\
\text { transcription real-time PCR }\end{array}$ & $\begin{array}{l}\text { Quantitative reverse } \\
\text { transcription real-time PCR }\end{array}$ & $\begin{array}{l}\text { Reverse transcription } \\
\text { recombinase- aided } \\
\text { amplification }\end{array}$ & Droplet digital reverse transcription PCR \\
\hline Targets & ORF1ab and $\mathrm{N}$ & ORF1ab & ORF1ab & ORF1ab and $\mathrm{N}$ \\
\hline $\begin{array}{l}\text { Internal } \\
\text { control }\end{array}$ & Yes & Yes & No & Yes \\
\hline $\begin{array}{l}\text { RNA load } \\
(\mu \mathrm{L})\end{array}$ & 5 & 10 & 5 & 15 \\
\hline $\begin{array}{l}\text { Limit of } \\
\text { detection } \\
\text { (copies/mL) }\end{array}$ & 1000 & 100 & 200 & 200 \\
\hline $\begin{array}{l}\text { Time spent } \\
\text { (min) }\end{array}$ & $\sim 87$ & 〜95 & $\sim 17$ & $\sim 160$ \\
\hline $\begin{array}{l}\text { Result } \\
\text { interpretation }\end{array}$ & $\begin{array}{l}\text { Positive: } \mathrm{Ct} \leq 38 \text {; Negative: } \\
\mathrm{Ct}>38 \text {; Retest: single gene } \\
\mathrm{Ct}>38 \text {. After retesting, double } \\
\text { genes } \mathrm{Ct} \leq 38 \text { for positive, } \\
\text { otherwise negative. }\end{array}$ & $\begin{array}{l}\text { Positive: } \mathrm{Ct} \leq 38 \text {; } \\
\text { Negative: No Ct; Retest: } \\
\text { Ct }>38 \text {. After retesting, the } \\
\text { sigmoidal curve e result is } \\
\text { considered positive. }\end{array}$ & $\begin{array}{l}\text { According to the } \\
\text { manufacturer's } \\
\text { instructions, set the } \\
\text { slope as } 20 \text { to } \\
\text { automatically judge the } \\
\text { test results. }\end{array}$ & $\begin{array}{l}\text { Positive: Copies of } O R F 1 a b \geq 3 \text { and } O R F 1 a b+N \geq 5 \text {, or } \mathrm{N} \geq 5 \text {; } \\
\text { Negative: } O R F 1 a b<3 \text {, and } O R F 1 a b+N<5 \text {; Retest: } O R F 1 a b<3 \text {, } \\
\text { and ORF1ab+N } \geq 5 \text {; After retesting, the suspicious result is } \\
\text { considered positive. The final viral RNA copy number was defined } \\
\text { as the higher value of the two genes. }\end{array}$ \\
\hline
\end{tabular}




\section{RESULTS}

\section{Patient Description}

The median age of the 72 patients in the study was 56 years (IQR $40-65$ years) and $62.5 \%$ of them were males. Fever (83.3\%), cough (54.2\%), and expectoration $(30.6 \%)$ were the most common clinical manifestations at the time of admission. 16 patients were admitted to the ICU, and 4 of them were under mechanical ventilation. $88.9 \%$ of patients received oxygen supplement. The demographic and clinical characteristics of the cross-section group $(n=68)$ and longitudinal group $(n=12)$ are shown in Table 2. Additionally, the median of days after symptoms onset of 68 patients in the cross-section group was 15 days (IQR, 11 19 days).

\section{Performance of the 4 Methods in Diluted Positive Samples}

The results showed that the performance of the four methods were different (Table 3). Specifically, in the testing of NPS, all four methods performed well with a dilution of 625 -fold. In the 3,125-fold diluted OPS and saliva sample, the performance of RT-PCR A was not as good as those of the other three methods.

\section{Comparison of the 4 Methods in the Test of Clinical Samples}

A total of 204 samples collected from 68 patients (all the 3 sample types were collected from each patient) were tested. The results showed that $55.9 \%(114 / 204)$ of the samples were positive by at least one of the 4 methods. The positivity rate of three methods (RT-PCR B, RT-RAA and dd-RT-PCR) were higher than $90 \%$ (91.2\%, 91.2\% and 93.0\%, respectively), while RT-PCR A showed the lowest positivity rate $(71.9 \%, 82 / 114)$. The positivity rate of dd-RT-PCR was the highest in every sample type (83.3\% in OPS, $97.6 \%$ in NPS, and $93.8 \%$ in saliva). If OPS samples were tested alone by RT-PCR A, only $21.4 \%$ (12/56) of the COVID-19 patients would be considered positive (Tables 4 and S1).

In addition, the performance of the 4 methods in testing 197 saliva samples from 12 patients who were sampled almost daily since admission were also compared. Among these samples, 166 (84.3\%) were SARS-CoV-2-positive by at least one of the 4 methods. The positivity rate of RT-PCR A, RT-PCR B, RTRAA, and dd-RT-PCR was $86.7 \%, 91.0 \%, 91.0 \%$ and $94.6 \%$, respectively (Tables $\mathbf{5}$ and $\mathbf{S 2}$ ).

\section{Effect of Sample Type on Detection Accuracy}

To determine the best sample type or combination of sample types in minimizing false negatives in the identification of SARSCoV-2 infection, we analyzed and compared the number of positive results detected by different methods using different sample types and sample combinations. Regardless of the method used, the positivity rate in testing saliva samples $(80.4 \% \sim 86.5 \%)$ was always the highest, followed NPS $(71.7 \% \sim 78.8 \%)$ and OPS $(26.1 \% \sim 38.5 \%)$. In contrast, the accuracy of the test can be improved to nearly $100 \%$ for each method when saliva and NPS from the same patient were tested simultaneously (Table 6).

TABLE 2 | Demographics and clinical characteristics of enrolled patients.

\begin{tabular}{|c|c|c|c|}
\hline \multirow[t]{2}{*}{ Variables } & \multicolumn{3}{|c|}{ No. of patients ( $\%$ of total) } \\
\hline & Total $(\mathrm{N}=72)$ & Cross-section group ${ }^{a}(\mathrm{~N}=68)$ & Longitudinal group $^{\mathrm{b}}(\mathrm{N}=12)$ \\
\hline \multicolumn{4}{|l|}{ Demographics } \\
\hline Median age(median [IQR]) (yr) & $56(40-65)$ & $54(40-64)$ & 55(39-65) \\
\hline Male sex & $45(62.5)$ & $41(60.3)$ & $8(66.7)$ \\
\hline \multicolumn{4}{|l|}{ Underlying disease } \\
\hline Hypertension & $25(34.7)$ & $22(32.4)$ & $6(50.0)$ \\
\hline Chronic heart disease & $3(4.2)$ & $3(4.4)$ & $0(0)$ \\
\hline Chronic lung disease & $6(8.3)$ & $6(8.8)$ & $2(16.7)$ \\
\hline Chronic liver disease & $3(4.2)$ & $3(4.4)$ & $1(8.3)$ \\
\hline Diabetes & $2(2.8)$ & $0(0)$ & $2(16.7)$ \\
\hline Solid tumor & $1(1.4)$ & $0(0)$ & $1(8.3)$ \\
\hline \multicolumn{4}{|l|}{ Symptoms } \\
\hline Fever & $60(83.3)$ & $56(82.4)$ & $10(83.3)$ \\
\hline Cough & $39(54.2)$ & $36(52.9)$ & $7(58.3)$ \\
\hline Sputum & $22(30.6)$ & 19(27.9) & $6(50.0)$ \\
\hline Chest distress & $7(9.7)$ & $7(10.3)$ & $O(0)$ \\
\hline Dizziness & $5(6.9)$ & $4(5.9)$ & $1(8.3)$ \\
\hline Headache & $3(4.2)$ & $3(4.4)$ & $1(8.3)$ \\
\hline Diarrhea & $8(11.1)$ & $8(11.8)$ & $1(8.3)$ \\
\hline Myalgia & $14(19.4)$ & $12(17.7)$ & 4(33.3) \\
\hline \multicolumn{4}{|l|}{ Disease severity } \\
\hline Oxygen supplement & $64(88.9)$ & $60(88.2)$ & $12(100)$ \\
\hline Invasive mechanical ventilation & $2(2.8)$ & 2(2.9) & $2(16.7)$ \\
\hline Intensive care unit admission & $16(22.2)$ & $12(17.7)$ & $5(41.7)$ \\
\hline
\end{tabular}

aSimultaneously collected OPS, NPS, and saliva samples from each hospitalized patient. Due to coma during sampling, 4 of the 72 patients were excluded;

${ }^{b} 12$ of the 72 hospitalized patients having well-preserved saliva after deep cough were enrolled in this study. 
TABLE 3 | Detection of SARS-CoV-2 in serial five-fold dilution of 3 samples.

\begin{tabular}{|c|c|c|c|c|c|c|}
\hline Sample & Dilution & \multicolumn{2}{|c|}{ RT-PCR A } & RT-PCR B ORF1ab (Ct) & RT-RAA ORF1ab (Min) & dd-RT-PCR ORF1ab and N (copies/test) \\
\hline \multirow[t]{5}{*}{ OPS } & $1 \times$ & 26.69 & 27.01 & 25.51 & 0.00 & 1476.8 \\
\hline & $25 x$ & 31.50 & 32.37 & 31.01 & 0.22 & 68.0 \\
\hline & $125 x$ & 33.71 & 35.20 & 33.21 & 0.89 & 9.3 \\
\hline & $625 x$ & $33.76^{\mathrm{a}}$ & $34.98^{\mathbf{a}}$ & 36.90 & 2.89 & 3.3 \\
\hline & $78,125 x$ & $\mathrm{~N}$ & N & $\mathrm{N}$ & $\mathrm{N}$ & $\mathrm{N}$ \\
\hline \multirow[t]{5}{*}{ NPS } & $1 \times$ & 27.29 & 28.04 & 26.71 & 0.00 & 12044.9 \\
\hline & $5 x$ & 28.83 & 29.55 & 28.93 & 0.00 & 585.6 \\
\hline & $25 x$ & 31.51 & 31.88 & 31.95 & 0.00 & 131.1 \\
\hline & $125 x$ & 33.37 & 33.82 & 33.41 & 0.33 & 29.3 \\
\hline & $625 x$ & 36.87 & 36.60 & 35.97 & 1.22 & 8.9 \\
\hline & $25 x$ & 26.72 & 27.83 & 27.36 & 0.00 & 1386.1 \\
\hline & $125 x$ & 29.19 & 31.00 & 31.25 & 0.00 & 131.6 \\
\hline & $625 x$ & 35.92 & 34.63 & 34.34 & 0.55 & 22.3 \\
\hline & $3,125 x$ & 37.22 & $36.81^{\mathbf{b}}$ & 37.10 & 2.78 & 5.2 \\
\hline & $15,625 x$ & N & $\mathrm{N}$ & N & $N$ & $1.0^{\mathrm{a}}$ \\
\hline & $78,125 x$ & $\mathrm{~N}$ & $\mathrm{~N}$ & $\mathrm{~N}$ & $\mathrm{~N}$ & $N$ \\
\hline
\end{tabular}

Bold values means that the corresponding dilution is the highest dilution.

${ }^{a}$ Two of three replicates were tested positive;

${ }^{b}$ One of three replicates was tested positive. $N$ represents three replicates negative;

${ }^{c}$ The final viral RNA copy number was defined as the higher value between the copy numbers of the two genes.

We further compared the absolute viral load in the 3 sample types of the 68 patients by dd-RT-PCR, and found that the median viral load of OPS (24.90 copies/test (IQR 15.58-129.85)) was significantly lower than that of NPS (274.40 copies/test (IQR 33.10-2836.45) and saliva (292.30 copies/test (IQR 20.208628.55) (Figure 2).

\section{Performance of the 4 Methods for Repeatedly Tested Patients}

Saliva samples from 12 patients who were sampled almost daily since admission were enrolled to dynamically evaluate the performance of the 4 methods. Among the 12 patients, samples of 5 patients were collected every day, while samples of 7 patients were missing on 1-2 days after onset. These patients were admitted to the hospital 3 14 days after the onset of the disease (Figure 3). We found that all the results were positive for the samples collected from most of the 12 patients within the first $1 \sim 2$ weeks after admission using four methods. During the later stages of the disease, the results of different methods varied. Only dd-RT-PCR produced positive results for the samples collected on days 21,22 , and 23 from patient 2.

\section{DISCUSSION}

Nucleic acid testing of SARS-CoV-2 is a vital basis for confirming the diagnosis of suspected COVID-19 patients and determining whether they should be quarantined or can be discharged. Currently, although various respiratory specimen types can be used for testing in the case of respiratory infections such as influenza and SARS-CoV-2, the positive rate of different sample types is different (Kim et al., 2017; To et al., 2019; Lin et al., 2020; Wang W. et al., 2020). And the false negative results of nucleic acid tests in clinical specimens have restricted rapid and accurate diagnosis. In many cases, repeated sampling must be required, combined with testing of different types of samples and clinical examination methods (such as computed tomography), to confirm the cases (Ai et al., 2020). Additionally, a large number of testing reagents are urgently needed to meet the needs of clinical screening and diagnosis, leading to the emergency usage of multiple newly developed methods which rarely have been tested before. Thus, selection of the most appropriate respiratory sample type, method and sample combination that affect the accuracy of nucleic acid detection are needed. And the clinical detection performance of these test kits or methods also needs to be objectively evaluated.

Our research showed that the detection abilities of different detection methods differed. The method with the highest positivity rate was dd-RT-PCR, followed by RT-RAA and RTPCR B, whereas RT-PCR A performed the worst. The dd-RTPCR method had the advantage of absolute quantification without need of the standards when compared to other RTPCR methods, as well as no amplification bias caused by interfering substances in the samples (Hindson et al., 2011; 
TABLE 4 | Performance of 4 methods in testing 204 samples from 68 patients.

\begin{tabular}{|c|c|c|c|c|c|c|}
\hline $\begin{array}{l}\text { Sample } \\
\text { type (n) }\end{array}$ & Method & $\begin{array}{l}\text { No. of positive } \\
\text { sample }\end{array}$ & $\begin{array}{l}\text { No. of positive sample by } \\
\text { any method }\end{array}$ & $\begin{array}{l}\text { Positivity rate } \\
{[\%(95 \% \mathrm{Cl})]}\end{array}$ & $\begin{array}{l}\text { No. of positive patients by any methods in } \\
\text { any sample types }\end{array}$ & $\begin{array}{l}\text { Positivity rate }^{\mathrm{b}} \\
{[\%(95 \% \mathrm{Cl})]}\end{array}$ \\
\hline \multirow[t]{4}{*}{ OPS (n=68) } & $\begin{array}{l}\text { qRT- } \\
\text { PCR A }\end{array}$ & 12 & 24 & 50.0 (29.6- 70.3) & $56^{\mathrm{C}}$ & $21.4(12.0-34.8)$ \\
\hline & $\begin{array}{l}\text { qRT- } \\
\text { PCR B }\end{array}$ & 18 & & 75.0 (52.9- 89.4) & & 32.1 (20.6- 46.1) \\
\hline & RT-RAA & 20 & & $83.3(61.8-94.5)$ & & $35.7(23.7-49.7)$ \\
\hline & $\begin{array}{l}\text { dd-RT- } \\
\text { PCR }\end{array}$ & 20 & & 83.3 (61.8- 94.5) & & $35.7(23.7-49.7)$ \\
\hline & $\begin{array}{l}\text { qRT- } \\
\text { PCR B }\end{array}$ & 41 & & 97.6 (85.9-99.9) & & $73.2(59.5-83.8)$ \\
\hline & RT-RAA & 39 & & $92.9(79.4-98.1)$ & & $69.6(55.7-80.8)$ \\
\hline & $\begin{array}{l}\text { dd-RT- } \\
\text { PCR }\end{array}$ & 41 & & 97.6 (85.9-99.9) & & $73.2(59.5-83.8)$ \\
\hline & RT-RAA & 45 & & 93.8 (81.8- 98.4) & & $80.4(67.2-89.3)$ \\
\hline & $\begin{array}{l}\text { dd-RT- } \\
\text { PCR }\end{array}$ & 45 & & 93.8 (81.8- 98.4) & & $80.4(67.2-89.3)$ \\
\hline \multirow[t]{4}{*}{$\begin{array}{l}\text { Total } \\
(n=204)\end{array}$} & $\begin{array}{l}\text { qRT- } \\
\text { PCR A }\end{array}$ & 82 & 114 & 71.9 (62.6- 79.7) & / & / \\
\hline & $\begin{array}{l}\text { qRT- } \\
\text { PCR B }\end{array}$ & 104 & & 91.2 (84.1-95.5) & / & / \\
\hline & RT-RAA & 104 & & $91.2(84.1-95.5)$ & / & / \\
\hline & $\begin{array}{l}\text { dd-RT- } \\
\text { PCR }\end{array}$ & 106 & & $93.0(86.2-96.7)$ & / & / \\
\hline
\end{tabular}

${ }^{a}$ No. of positive sample/No. of positive sample by any method.

${ }^{b}$ No. of positive sample/No. of positive patients by any methods in any sample types.

${ }^{c}$ The remaining 12 confirmed patients were negative by any methods in any sample types in sampling day.

Li et al., 2018). Suo et al. also found that the sensitivity was improved from $40 \%$ for RT-PCR to $94 \%$ for dd-RT-PCR in SARS-CoV-2 detection on throat swab of 63 suspected patients and 14 supposed convalescents (Suo et al., 2020). According to our results, dd-RT-PCR performed better in detecting low-viralload samples in the late stage of disease and reduced the false negative reports, which could be a powerful complement to the RT-PCR. RT-RAA is simple and time-saving with credible sensitivity and specificity. Furthermore, minimal requirements for equipment and resources make it especially suitable for onsite inspection in the underdeveloped areas, which has broad

TABLE 5 | Performance characteristics of 4 methods in testing 197 saliva samples from 12 patients.

\begin{tabular}{lccc}
\hline Method & $\begin{array}{c}\text { No. of positive } \\
\text { samples }\end{array}$ & $\begin{array}{c}\text { No. of positive samples } \\
\text { by any method }\end{array}$ & $\begin{array}{c}\text { Positivity rate }^{\mathbf{a}} \\
(\%[95 \% \text { Cl] })\end{array}$ \\
\hline $\begin{array}{l}\text { RT-PCR } \\
\text { A }\end{array}$ & 144 & 166 & $86.7(80.4-91.3)$ \\
RT-PCR & 151 & $91.0(85.2-94.7)$ \\
B & & $91.0(85.2-94.7)$ \\
RT-RAA & 151 & $94.6(89.6-97.3)$ \\
dd-RT- & 157 & \\
PCR & &
\end{tabular}

a Number of positive samples divided by the number of positive samples by any method. application prospects ( $\mathrm{Li}$ et al., 2020). It was reported that the established POCT assay-based RAA offered $100 \%$ specificity and $100 \%$ sensitivity in the detection of clinical respiratory tract samples from COVID-19 patients when compared with RT-PCR (Xue et al., 2020; Zheng et al., 2021). Additionally, a multiplecenter clinical evaluation of RT-RAA kit using respiratory tract samples (throat swabs, sputum, nasopharyngeal swabs, nasal swabs and bronchoalveolar lavage fluid) and non-respiratory samples (stool and whole blood) show that the total coincidence rate was $97.78 \%$ and the kappa value $0.952(\mathrm{p}<0.05)$ compared to the commercial RT-PCR kits (Wang J. et al., 2020). In the present study, the positivity rate of RT-RAA was second only to dd-RT-PCR and nearly the same as RT-PCR B. It is concluded that RT-RAA is a reliable method worthy of promotion. RT-PCR is the most widely used method for respiratory virus detection. In our study, the positivity rate of RT-PCR A was lower than that of RT-PCR B, even though RT-PCR A is a two-gene (ORF1ab and N) testing, while RT-PCR B only tests one gene (ORF1ab). Primer design may be one of the problems, which is the most common cause of false negatives in RT-PCR (Jung et al., 2020). Therefore, to minimize false negatives, laboratories should choose the nucleic acid detection method with the higher positivity rate according to the laboratory conditions.

In our study, four methods were used for simultaneous testing, and the gold standard for diagnosis was any positive 
TABLE 6 | Number of positive results detected by different methods using different sample types and sample combinations.

Sample types or combinations

RT-PCR A

OPS

NPS

Saliva

OPS + NPS

OPS+ Saliva ${ }^{\mathrm{C}}$

NPS+ Saliva ${ }^{\mathrm{C}}$

OPS + NPS+ Saliva
No. of positive sample types or combinations, $\mathrm{n}\left(\%^{\mathrm{a}}\right)$

\begin{tabular}{llcc} 
RT-PCR B & RT-RAA & dd-RT-PCR & any method $^{\mathbf{b}}$ \\
\hline $18(34.6)$ & $20(38.5)$ & $20(37.7)$ & $24(42.9)$ \\
$41(78.8)$ & $39(75.0)$ & $41(77.4)$ & $42(75.0)$ \\
$45(86.5)$ & $45(86.5)$ & $45(84.9)$ & $48(85.7)$ \\
$41(78.8)$ & $41(78.8)$ & $43(81.1)$ & $46(82.1)$ \\
$45(86.5)$ & $45(86.5)$ & $46(86.8)$ & $49(87.5)$ \\
$52(100)$ & $52(100)$ & $52(98.1)$ & $55(98.2)$ \\
$52(100)$ & $52(100)$ & $53(100)$ & $56(100)$
\end{tabular}

${ }^{a}$ The number of positive samples of each sample type or sample combination divided by the total number of positive samples.

${ }^{b}$ Number of positive samples by any method.

${ }^{c}$ The combined sample was considered positive if any of the two or three samples from the same patient was positive.

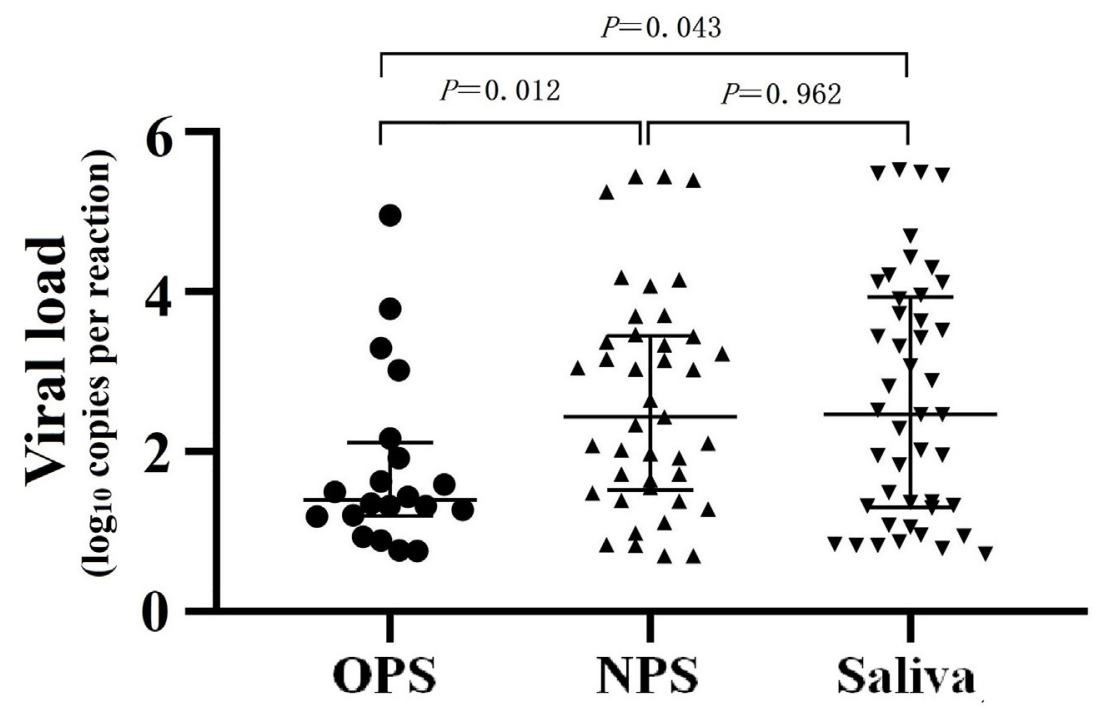

FIGURE 2 | SARS-CoV-2 viral load in different sample types detected by dd-RT-PCR.

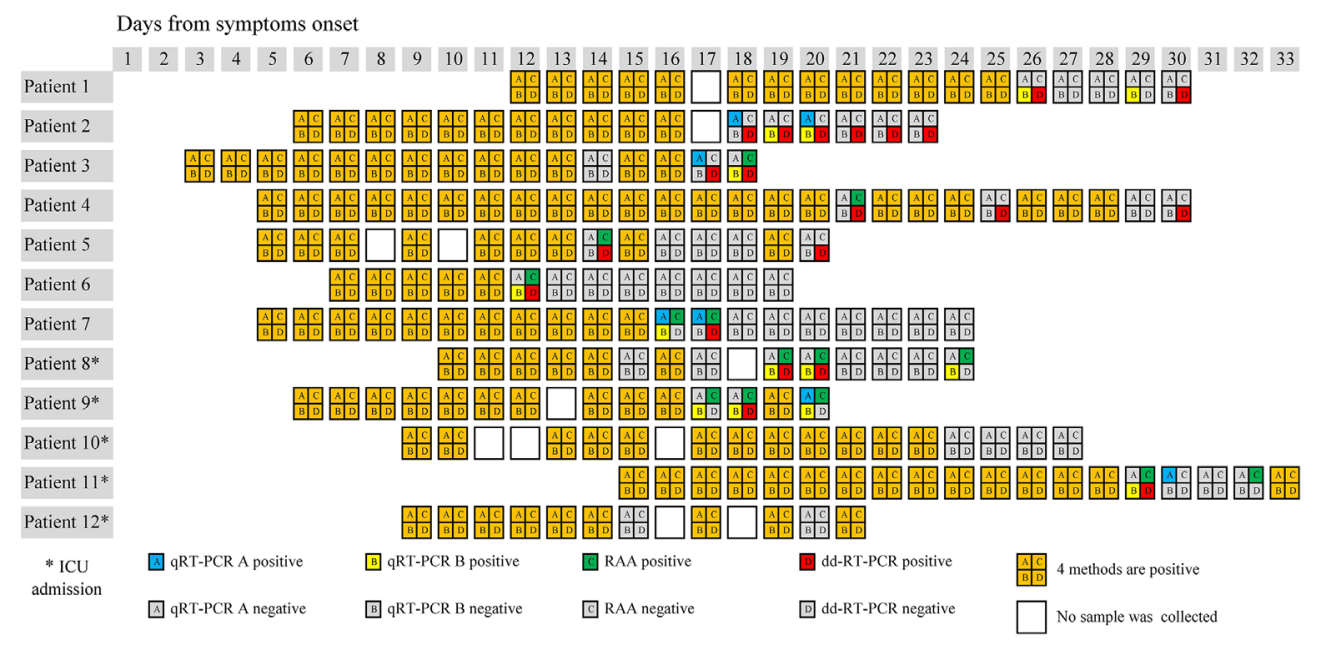

FIGURE 3 | Results of the 4 methods in the testing of 197 saliva samples of 12 patients after admission. 
result of the four methods. The results demonstrated that saliva specimens showed higher positivity rate than NPS and OPS and supported the findings of previous studies (Fan et al., 2020; To et al., 2020; Sakanashi et al., 2021). It may be attributed to high expression of ACE2 in the alveoli, which is the receptor of SARS$\mathrm{CoV}-2$, making a large amount of virus accumulate in the lower respiratory tract (Fan et al., 2020; Wan et al., 2020). After a deep cough, the virus is flushed out with air pressure and wrapped in sputum or saliva, thereby making saliva to contain a higher viral load and easier to be detected positive (Fan et al., 2020; Wan et al., 2020). A recent study in Hong Kong found that the median load of SARS-CoV-2 in saliva can reach $3.3 \times 10^{6}$ copies $/ \mathrm{mL}$ (range from $9.9 \times 10^{2}$ to $1.2 \times 10^{8}$ copies $/ \mathrm{mL}$ ) (To et al., 2020). Some other studies have shown that the concentration of SARSCoV-2 in NPS is higher than that in OPS (Zou et al., 2020). However, the collection of NPS is relatively complicated and causes significant discomfort to patients and is associated with infection risk to healthcare workers (Frazee et al., 2018; Ai et al., 2020). Due to the fast and convenient collection of OPS, it has become the first choice of many clinics. Consistent with some studies (Fan et al., 2020; Lin et al., 2020), the detection rate of OPS was as low as $42.9 \%$ in our study. And the absolute quantitative results of dd-RT-PCR also showed that the viral load in OPS was much lower than that in other type of samples, indicating a high rate of missed detection when testing samples with low viral loads. Besides, we also found that about four-fifths of the positive COVID-19 patients would be missed when detecting positive patients' OPS using RT-PCR A, which raised serious risk for SARS-CoV-2 transmission. Therefore, it is prompted that use of inappropriate sample types was the most important reason for missed detection. According to our results, saliva after deep cough can be collected by patients themselves in a noninvasive manner, which is suitable for nucleic acid detection of SARS-CoV-2. The sample collection principle is to wear a mask and deep cough 3 to 5 times, and then immediately open the sterile container behind the mask and spit out saliva. There are several advantages of using saliva after deep cough for clinical detection, including better patient compliance, simple operation, reduced risk of medical staff infection and overcoming the shortage of personal protective equipment and specimen sampling tools. However, it should be noted that false negatives may still occur even when using saliva samples. As shown in Figure 2, a negative result suddenly appeared one day during a patient's continued positive phase, such as patient 3 on day 14, patient 8 on day 15 , and patient 12 on day 15 . According to these results, we found that supplemental NPS testing can make up for missed detection of saliva samples. Taken together, for highly suspected or confirmed patients before discharging from the hospital, saliva after deep cough can be collected as well as NPS for simultaneous testing. We also recommend adding the evaluation of saliva during registration evaluation of SARSCoV-2 nucleic acid detection reagents.

We acknowledge our limitations. Firstly, all patients enrolled in our study are confirmed COVID-19 patients, while samples of non-confirmed patients or patients with other respiratory tract pathogenic infections were not included. And the sample was considered true positive if it was positive detected by any method. Thus, it is difficult to analyze the sensitivity and specificity of different methodologies in this study. Secondly, given that COVID-19 patients mostly have dry cough with less sputum, we require that all patients wear masks and deep cough $3 \sim 5$ times before spitting saliva into a sterile container when sampled. We called the sample "saliva after deep cough". By this new sampling method, almost all patients are able to collect this specimen by themselves, except some patients who are unconscious. Thus, it was indeed difficult to distinguish sputum from saliva samples. Finally, this study was a single center cohort study and only samples from hospitalized patients were enrolled, which could lead to an unbalanced distribution of confounders when evaluating the positivity rate of different methods.

In conclusion, saliva after deep cough is preferred for the detection of SARS-CoV-2 and NPS should be collected for simultaneous testing if necessary. It is not recommended to only collect OPS for SARS-CoV-2 detection. Furthermore, laboratories should fully evaluate the nucleic acid detection methods, and highly sensitive methods like dd-RT-PCR are recommended to reduce missed diagnosis.

\section{DATA AVAILABILITY STATEMENT}

The raw data supporting the conclusions of this article will be made available by the authors, without undue reservation.

\section{ETHICS STATEMENT}

The studies involving human participants were reviewed and approved by Clinical Research Ethics Committee of the First Affiliated Hospital, College of Medicine, Zhejiang University. Written informed consent for participation was not required for this study in accordance with the national legislation and the institutional requirements.

\section{AUTHOR CONTRIBUTIONS}

YC, BL, and FY designed the study. GX, FY, DZ, BF, QW, QZ, RW, XY and WC performed the experiments. FY, GX, SZ and $\mathrm{DH}$ analyzed the data. FY, GX, SZ, DH and JB wrote the manuscript. All authors contributed to the article and approved the submitted version.

\section{FUNDING}

This work was supported by the National Natural Science Foundation of China (grant numbers 81803290, 81971919 and 
82072377), National Key R\&D Program of China (2020YFC0847800 and 2020YFC0848000) and Department of Education of Zhejiang Province (No. Y202043387).

\section{ACKNOWLEDGMENTS}

We acknowledge the contributions of other clinical and technical staff of the First Affiliated Hospital, College of Medicine,

\section{REFERENCES}

Ai, T., Yang, Z., Hou, H., Zhan, C., Chen, C., Lv, W., et al. (2020). Correlation of Chest CT and RT-PCR Testing for Coronavirus Disease 2019 (Covid-19) in China: A Report of 1014 Cases. Radiology 296, E32-E40. doi: 10.1148/ radiol.2020200642

Axell-House, D. B., Lavingia, R., Rafferty, M., Clark, E., Amirian, E. S., and Chiao, E. Y. (2020). The Estimation of Diagnostic Accuracy of Tests for COVID-19: A Scoping Review. J. Infect. 81, 681-697. doi: 10.1016/j.jinf.2020.08.043

Chu, D., Pan, Y., Cheng, S., Hui, K., Krishnan, P., Liu, Y., et al. (2020). Molecular Diagnosis of a Novel Coronavirus, (2019-Ncov) Causing an Outbreak of Pneumonia. Clin. Chem. 66, 549-555. doi: 10.1093/clinchem/hvaa029

Fan, J., Yu, F., Wang, X., Zou, Q., Lou, B., Xie, G., et al. (2020). Hock-a-Loogie Saliva as a Diagnostic Specimen for SARS-CoV-2 by a PCR-based Assay: A Diagnostic Validity Study. ClinChimActa 511, 177-180. doi: 10.1016/j.cca. 2020.10.004

Fang, Y., Zhang, H., Xie, J., Lin, M., Ying, L., Pang, P., et al. (2020). Sensitivity of Chest CT for COVID-19: Comparison to RT-PCR. Radiology 296, E115-E117. doi: $10.1148 /$ radiol.2020200432

Frazee, B. W., Rodriguez-Hoces, D. L. G. A., Alter, H., Chen, C. G., Fuentes, E. L., Holzer, A. K., et al. (2018). Accuracy and Discomfort of Different Types of Intranasal Specimen Collection Methods for Molecular Influenza Testing in Emergency Department Patients. Ann. Emerg. Med. 71, 509-517. doi: 10.1016/ j.annemergmed.2017.09.010

Hindson, B. J., Ness, K. D., Masquelier, D. A., Belgrader, P., Heredia, N. J., Makarewicz, A. J., et al. (2011). High-Throughput Droplet Digital PCR System for Absolute Quantitation of DNA Copy Number. Anal Chem 83, 8604-8610. doi: $10.1021 / \mathrm{ac} 202028 \mathrm{~g}$

Jung, Y., Park, G. S., Moon, J. H., Ku, K., Beak, S. H., Pang, P., et al. (2020). Comparative Analysis of Primer-Probe Sets for RT-qPCR of COVID-19 Causative Virus (Sars-Cov-2). ACS Infect. Dis. 6, 2513-2523. doi: 10.1021/acsinfecdis.0c00464

Kim, Y. G., Yun, S. G., Kim, M. Y., Park, K., Cho, C. H., et al. (2017). Comparison Between Saliva and Nasopharyngeal Swab Specimens for Detection of Respiratory Viruses by Multiplex Reverse Transcription-Pcr. J. ClinMicrobiol 55, 226-233. doi: 10.1128/JCM.01704-16

Li, H., Bai, R., Zhao, Z., Tao, L., Ma, M., Lee, C. S., et al. (2018). Application of Droplet Digital PCR to Detect the Pathogens of Infectious Diseases. Biosci. Rep. 38. doi: 10.1042/BSR20181170

Li, J., Macdonald, J., and von Stetten, F. (2020). Correction: Review: A Comprehensive Summary of a Decade Development of the Recombinase Polymerase Amplification. Analyst 145, 1950-1960. doi: 10.1039/c9an90127b

Lin, C., Xiang, J., Yan, M., Li, H., Huang, S., and Shen, C. (2020). Comparison of Throat Swabs and Sputum Specimens for Viral Nucleic Acid Detection in 52 Cases of Novel Coronavirus (SARS-Cov-2)-Infected Pneumonia (Covid-19). Clin. Chem. Lab. Med. 58, 1089-1094. doi: 10.1016/j.jiac.2020.09.027

Sakanashi, D., Asai, N., Nakamura, A., Miyazaki, N., Kawamoto, Y., Ohno, T., et al. (2021). Comparative Evaluation of Nasopharyngeal Swab and Saliva Specimens for the Molecular Detection of SARS-CoV-2 RNA in Japanese Patients With COVID-19. J Infect Chemother 27, 126-129. doi: 10.1016/j.jiac.2020.09.027
Zhejiang University; and Prof. Yong Guo from the Tsinghua University for comments on the manuscript.

\section{SUPPLEMENTARY MATERIAL}

The Supplementary Material for this article can be found online at: https://www.frontiersin.org/articles/10.3389/fcimb.2021. 685640/full\#supplementary-material

Suo, T., Liu, X., Feng, J., Guo, M., Hu, W., Guo, D., et al. (2020). Ddpcr: A More Accurate Tool for SARS-CoV-2 Detection in Low Viral Load Specimens. Emerg. Microbes Infect. 9, 1259-1268. doi: 10.1080/22221751.2020.1772678

To, K. K., Tsang, O. T., Yip, C. C., Chan, K. H., Wu, T. C., Chan, J. M., et al. (2020). Consistent Detection of 2019 Novel Coronavirus in Saliva. Clin. Infect. Dis. 71, 841-843. doi: 10.1093/cid/ciaa149

To, K., Yip, C., Lai, C., Wong, C., Ho, D., Pang, P., et al. (2019). Saliva as a Diagnostic Specimen for Testing Respiratory Virus by a Point-of-Care Molecular Assay: A Diagnostic Validity Study. Clin. Microbiol. Infect. 25, 372-378. doi: 10.1016/j.cmi.2018.06.009

Wan, Y., Shang, J., Graham, R., Baric, R. S., and Li, F. (2020). Receptor Recognition by the Novel Coronavirus From Wuhan: An Analysis Based on Decade-Long Structural Studies of SARS Coronavirus. J. Virol. 94 (7), e00127-20. doi: 10.1128/JVI.00127-20

Wang, J., Cai, K., He, X., Shen, X., Wang, J., Liu, J., et al. (2020). Multiple-Centre Clinical Evaluation of an Ultrafast Single-Tube Assay for SARS-CoV-2 Rna. Clin. Microbiol. Infect. 26, 1076-1081. doi: 10.1016/j.cmi.2020.05.007

Wang, W., Xu, Y., Gao, R., Lu, R., Han, K., Wu, G., et al. (2020). Detection of SARS-CoV-2 in Different Types of Clinical Specimens. Jama 323, 1843-1844. doi: $10.1001 /$ jama.2020.3786

World Health Organization (2021). Coronavirus Disease 2019 (Covid-19). Available at: https://www.who.int/emergencies/diseases/novel-coronavirus2019 [Accessed May 11, 2021].

Xue, G., Li, S., Zhang, W., Du, B., Cui, J., Yan, C., et al. (2020). ReverseTranscription Recombinase-Aided Amplification Assay for Rapid Detection of the 2019 Novel Coronavirus (Sars-Cov-2). Anal. Chem. 92, 9699-9705. doi: 10.1021/acs.analchem.0c01032

Zhang, X., Guo, L., Ma, R., Cong, L., Wu, Z., Wei, Y., et al. (2017). Rapid Detection of Salmonella With Recombinase Aided Amplification. J. Microbiol. Methods 139, 202-204. doi: 10.1016/j.mimet.2017.06.011

Zheng, Y. Z., Chen, J. T., Li, J., Wu, X. J., Wen, J. Z., Liu, X. Z., et al. (2021). Reverse Transcription Recombinase-Aided Amplification Assay With Lateral Flow Dipstick Assay for Rapid Detection of 2019 Novel Coronavirus. Front. Cell Infect. Microbiol. 11, 613304. doi: 10.3389/fcimb.2021.613304

Zou, L., Ruan, F., Huang, M., Liang, L., Huang, H., Hong, Z., et al. (2020). SarsCov-2 Viral Load in Upper Respiratory Specimens of Infected Patients. N Engl. J. Med. 382, 1177-1179. doi: 10.1056/NEJMc2001737

Conflict of Interest: The authors declare that the research was conducted in the absence of any commercial or financial relationships that could be construed as a potential conflict of interest.

Copyright (c) 2021 Yu, Xie, Zheng, Han, Bao, Zhang, Feng, Wang, Zou, Wang, Yang, Chen, Lou and Chen. This is an open-access article distributed under the terms of the Creative Commons Attribution License (CC BY). The use, distribution or reproduction in other forums is permitted, provided the original author(s) and the copyright owner(s) are credited and that the original publication in this journal is cited, in accordance with accepted academic practice. No use, distribution or reproduction is permitted which does not comply with these terms. 